\title{
Multi-spectral characterization of solar gamma ray events radio emission. Study case July 14, 2000.
}

\author{
Rodriguez Taboada R.E. ${ }^{1}$ and Mendez Berhondo A.L. ${ }^{1}$ \\ ${ }^{1}$ Institute of Geophysics and Astronomy, Calle 212 No 2906, La Coronela, C. Habana, Cuba \\ CP 11600 e-mail: ramone@infomed.sld.cu
}

\begin{abstract}
Solar sporadic radio emission takes place in a wide range of time scales; from milliseconds to days. Researches have been conduced mainly to explain the behavior of radio emission in specific time scales and lately to millisecond spikes research has been devoted a lot of papers.

In this work we pay attention to the characteristics of millisecond spikes in relation to the general activity in which they are included, processes with very different time scales.
\end{abstract}

\section{Data processing and results}

Radio polarimeter observations in 237, 327, 408, 610, 1420 and $2695 \mathrm{MHz}$ with a digitalization rate of $100 \mathrm{~Hz}$ were provided by the Trieste Astronomical Observatory (OAT). Some complementary data were obtained from open sources in Internet (Goes $\mathrm{X}-\mathrm{R}$, SOHO images, etc.) Analyzed data intervals were selected in different evolutionary stages and locations in the $\mathrm{R}$ vs. $\mathrm{L}$ circular polarization diagrams of the whole event. Once an interval was selected it was processed in all frequencies.

Considering the event has two main activity periods, the interval between them diminishes with decreasing frequency. To judge on the flux spectrum of the activity is difficult because of the appearance of peaks not noticed in all frequencies. The $\mathrm{R}$ vs. $\mathrm{L}$ circular polarization diagrams (see Fig. 1) show the event goes from a simple linear relationship pointing to a homogeneous source in $2695 \mathrm{MHz}$ to a complex structure dominated by three main branches over which the spike activity takes places (noticeable clear in 610 $\mathrm{MHz}$ ).

The power spectra of 2000 data samples of selected intervals were calculated for L and $\mathrm{R}$ components and the temporal evolution of the power of the fluctuations resume. In any of the selected samples there is no evidence for a significant contribution of spikes to the overall energy release.

Considering the importance of circular polarization to analyze the generation mechanism (Mosunov A.N. and Charikov Yu. E., 1995, Astron. Zhurnal, 72, 911), individual spikes well identified in different intervals were used to measure spike profiles and no significant difference between rise and fall characteristic times with the evolutionary stage of the event found. Probably a higher resolution need. 

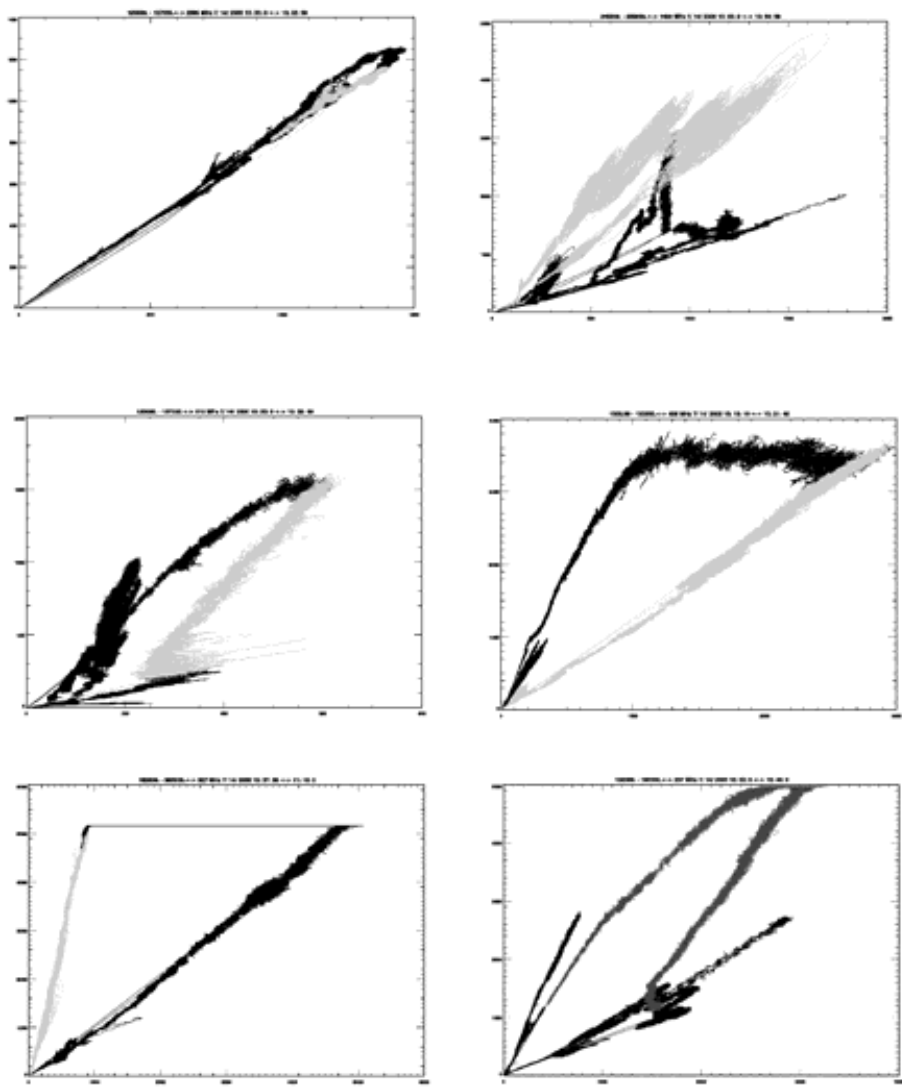

Figure 1. Polarization diagrams - up to down, from left to right, 2695, 1420, 610, 408, 327 and $237 \mathrm{MHz}$. In the lower corona $(2695 \mathrm{MHz})$ the diagram is simple, but in $1420 \mathrm{MHz}$ it is possible to see a much more complex structure. The initial linear branch (lower in black) with a superimposed structure related to the appearance of a source (mainly right polarized) and in gray the section related to a second activity period not visible on $2965 \mathrm{MHz}$. In 408 and 327 $\mathrm{MHz}$ the structure appears to be quite similar (saturated register limits the comparison), and in $237 \mathrm{MHz}$ the maximum of the event is marked in red. The $610 \mathrm{MHz}$ diagram (note it resembles more the $237 \mathrm{MHz}$ than the $408 \mathrm{MHz}$ diagram shows clearly that the spike activity conserves the characteristics of the "initial" source. 